\title{
Friction predication on pin-to-plate interface of PTFE material and steel
}

\author{
Zhuming BI ${ }^{1,2, *}$, Donald W. MUELLER ${ }^{1}$ \\ ${ }^{1}$ Depatment of Civil and Mechnical Engineering, Purdue University Fort Wayne, Fort Wayne 2101E, USA \\ ${ }^{2}$ School of Mechanical and Electrical Engineering, GuangDong University of Technology, Guangzhou 510006, China \\ Received: 07 February 2018 / Revised: 12 April 2018 / Accepted: 26 May 2018 \\ (C) The author(s) 2018. This article is published with open access at Springerlink.com
}

\begin{abstract}
In this paper, the friction behavior at a pin-to-plate interface is investigated. The pin and plate are made of Polytetrafluoroethylene (PTFE) and steel, respectively, and there is a reciprocating motion at the interface. Governing mathematical models for the relations of design variables and frictions are investigated, and a general procedure is proposed to solve the developed models and predict the friction forces at the interface subjected to given test conditions. Novel models have been developed to represent intrigued friction behaviors affected by various factors such as pin geometrics and finishes, lubrication conditions, and reciprocating speed. The test data from experiments is used to verify the effectiveness of the proposed models.
\end{abstract}

Keywords: pin-to-plate; friction; wear; coefficient of frictions; PTFE; sealing; finite element analysis

\section{Introduction}

The loss of the annual revenue due to friction and wear related problems is estimated at $6 \%$ of US GNP, which corresponds to almost $\$ 20$ billion [1]. Friction not only reduces the efficiency of power transmission, but also generates heat, raises temperature, and weakens the strength of components in most occasions. It is desirable to reduce the friction in many applications. A machine or machine tool generally involves in a number of relative motions among a group of components; to reduce adverse factors of friction, lubrication is often applied and sealing parts would be needed at interacting surfaces of contacts [2, 3]. Moreover, the friction behaviors directly relate to the wastes of lubrication and the shortage of product lifecycle. Design optimization of sealing parts from the perspective of wastes is critical to achieve the sustainability of manufacturing systems [4-6]. Note that friction behavior is really complicated; it is related to many factors such as material properties, surface conditions, lubrications, loads and operation conditions. Friction has been classified into dry friction, mixed boundary friction, and fully hydrodynamic friction. Among these types, a fully hydrodynamic friction is often desired since it has a low friction. The study of the friction and wear of machines has been a classic research subject, which attracts a great deal of attention. Recent research progress in this field has been reported by Refs. [7-11]. However, most of the researches are tied to specific products. For example, the friction on piezoelectricdriven stick-slip actuators was investigated by Zhang et al. [8, 9] and Liu e al. [11]. Without losing the generality, we are especially interested in the possibility of a critical change of pin geometry, which leads to a transition of the types of friction behavior such as the transition from dry friction and mix friction, or the mix friction and full hydrodynamic friction.

\subsection{PTFE composite and its material properties}

The friction coefficient relates to materials properties of objects as well as the contact conditions at interfaces. In this paper, the friction between PTFE and the

* Corresponding author: Zhuming BI, E-mail: biz@pfw.edu 
reciprocating plate is focused. PTFE is a type of widely used industrial materials. One important application is as bearing materials since PTFE exhibits a lower coefficient of friction compared to other polymers. PTFE has higher elastic modulus and better thermal resistance. Table 1 provides the material properties of PTFE from different sources [12-14].

Note that the material properties such as coefficient of friction (COF) vary from one contact condition to another. For example, ZEUS [15] suggested that the COF ranged from 0.9 to 1.0 under a static condition, from 0.01 to 0.1 for a partial lubrication, and from 0.001 to 0.01 for a full hydrodynamic lubrication. Biswas et al. [16] provided the test data showing that COF was in a range of 0.2 to 0.4 under the roughness of $0.3 \mu \mathrm{m}$ and a varying velocity from 0.14 to $1.17 \mathrm{~m} / \mathrm{s}$. The change of PTFE ingredients can certainly change the properties of PTFE composites. For example, Xie et al. [17] found that Potassium Titanate Whiskers (PTW) improved tribological properties significantly, this was based on testing of the pin-on-disk configuration under dry sliding condition at different applied loads.

Sonne et al. [18] investigated the combination of constitutive model and contact behavior, that was required to obtain accurate simulation of mechanical deformations of PTFE materials. PTFE exhibited a low coefficient of friction from 0.04 to 0.10 at large scales and typical contact conditions. However, when going down in contact faces and scales, the value of friction

Table 1 Materials properties of PTFE.

\begin{tabular}{ccc}
\hline Property & Units & Value \\
\hline Specific gravity & $\mathrm{g} / \mathrm{cm}^{3}$ & $2.0 / 4.0$ \\
Hardness & Type D & $55 / 65$ \\
Tensile strength & $\mathrm{MPa}$ & 15 minimum \\
Compressive strength & $\mathrm{MPa}(0.2$ Offset $)$ & 8.2 \\
& $\mathrm{MPa}(1 \%$ Offset $)$ & 5.8 \\
& $\mathrm{MPa}(5 \%$ Offset $)$ & 13.9 \\
Thermal coefficient & $\mu \mathrm{m} /\left(\mathrm{m} \cdot{ }^{\circ} \mathrm{C}\right)($ in the range of & 138.4 \\
Thermal conductivity & $25^{\circ} \mathrm{C}$ to $\left.100{ }^{\circ} \mathrm{C}\right)$ & \\
Young's modulus & $\mathrm{W} /(\mathrm{m} \cdot \mathrm{K})$ & 0.28 \\
Poisson ratio & $\mathrm{MPa}$ & 653.2 \\
Shear strength & & 0.46 \\
Friction of coefficient & $\mathrm{MPa}$ & 5 \\
\hline
\end{tabular}

coefficient was changed drastically. Researchers agreed that the coefficient of friction is increased when the normal pressure between two contact surfaces decreases. For example, at a normal pressure of $0.1 \mathrm{MPa}$, the friction coefficient between steel and PTFE was found as high as 0.4 .

\subsection{Studies on friction}

The studies on friction and wear have been mostly related to the seals at the contacts with a relative motion. Seals are important components to ensure the lives of machine elements. Seals are designed to prevent leakages and protect machine elements from the contamination. In particular, the behavior of radial lip seals was extensively studied in late 1960s. Jagger [19] firstly found the existence of the lubrication film under the lip of seal. Other suggested the micro texture of rubber surface played an important role in the film formation.

Characteristics of material friction depend on various factors. Kato [20] argued that the coefficients of friction and wear should not be treated as material properties-they should be a response of a tribosystem. A number of friction models are available. For example, Quaglini et al. [21] found that friction of polymers on smooth metal surfaces is characterized by two regions based on normal load: (1) with low sensitivity at low stress levels and (2) a sharp change in the rate of decrease of friction with increasing pressure above the plastic flow limit of the polymer.

To reduce friction, a fully hydrodynamic lubrication is desirable. Stolarshi [22] indicated that a necessary pressure of hydrodynamic lubrication depends on the gradient of the velocity profile along the film thickness. Three common methods to achieve this goal are: (1) externally pressurized lubrication - use a pump to force fluid flow to the center of the bearing and distribute it to outward through narrow space between parallel surfaces; (2) squeeze-film lubricationmove one surface normal to the other surface rapidly, with the viscous resistance to the displacement of oil; and (3) wedge-film lubrication-place one surface so that it is slightly inclined to the other and slide each other; lubricant is dragged into the converging space between them. The second method is specially considered in this report. 
Friction depends greatly on pressure, and it is crucial to understand the pressure distributions under given certain loads, part geometry, surface condition, and fluid flow. Stolarshi et al. [22] derived the governing equations of fluid dynamics as below.

As shown in Fig. 1, let a thin film exist between the two moving surfaces 1 and 2. The former flat is lying in the $X-Z$ plane, the latter curved and inclined. Component velocities $U, V$ and $W$ exist in the directions of $X, Y$ and $Z$, respectively. At any instant, two points having the same $x, z$ coordinates and separated by a distance $h$ will have absolute velocities which give the following set of boundary conditions:

$$
\left.\begin{array}{llll}
y=0, & u=U_{1}, & v=V_{1}, & w=W_{1} ; \\
y=h, & u=U_{2}, & v=V_{2}, & w=W_{2} .
\end{array}\right\}
$$

The pressure gradients $\partial p / \partial x$ and $\partial p / \partial z$ are independent of $y$ in a thin film, and $\partial p / \partial y=0$. The mass conservation of an incompressive fluid leads to,

$$
\begin{gathered}
\frac{1}{6}\left(\frac{\partial}{\partial x}\left(\frac{h^{3}}{\mu} \frac{\partial p}{\partial x}\right)+\frac{\partial}{\partial z}\left(\frac{h^{3}}{\mu} \frac{\partial p}{\partial z}\right)\right)=\left(U_{1}-U_{2}\right) \frac{\partial h}{\partial x}-2\left(V_{1}-V_{2}\right) \\
\quad+\left(W_{1}-W_{2}\right) \frac{\partial h}{\partial z}+h \frac{\partial}{\partial x}\left(U_{1}+U_{2}\right)+h \frac{\partial}{\partial z}\left(W_{1}+W_{2}\right)
\end{gathered}
$$

The last two terms are insignificant since the surface velocities $U$ and $W$ rarely change. The variants of Eq. (1) with slight differences can be found in many other literatures [23-26]. For example, Salant et al. [23] demonstrated that small fluctuations on the shaft surface can produce large hydrodynamic effects in the sealing zone due to the non-linearity of the Reynolds equation. The considered factors include surface profiles, roughness height and wavelength, lead angle

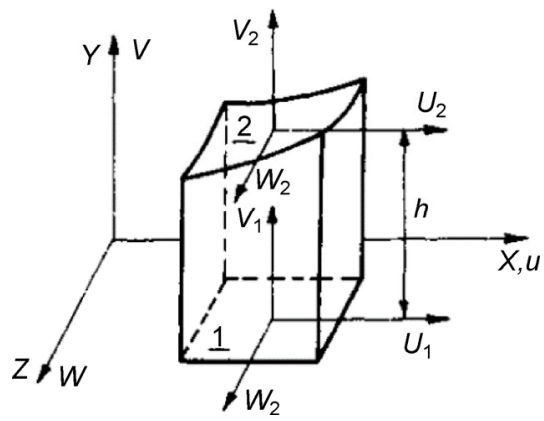

Fig. 1 Wedge-film lubrication. on load support, reverse pumping rate and cavitation area. The governing equation applied was

$$
\begin{aligned}
& \frac{\partial}{\partial x}\left[h^{3} \frac{\partial S \varphi}{\partial x}\right]+\Lambda^{2} \frac{\partial}{\partial y}\left[h^{3} \frac{\partial S \varphi}{\partial y}\right]=\gamma \frac{\partial\{[1+(1-S) \varphi] h\}}{\partial x} \\
& \quad+2 \gamma \frac{\partial\{[1+(1-S) \varphi] h\}}{\partial t}
\end{aligned}
$$

where

$$
\begin{array}{ll}
p=S \varphi & \\
S=1 & \varphi \geq 0 ; \\
S=0 & \varphi<0 .
\end{array}
$$

In their study, the Reynold's equation was decoupled by selecting an explicit expression for $h$ based on previous numerical and experimental results. The film thickness, averaged over the fluctuations due to the asperities, is relatively uniform and on the order of a micron. It is assumed that the film thickness equals to a constant plus a fluctuating term due to the asperities on the lip and a fluctuating term due to the shaft surface finish.

The friction behavior of material depends closely on the surface conditions of two contact areas. According to Bowden and Tabor, the surfaces would touch at the highest asperity peak firstly on each other, at this point the contact pressure would be so great that the peak would deform plastically until several other peaks touched. This would continue until enough peaks were contacting to support the normal load placed on the contact [27].

\subsection{Experimental approaches}

Due to the complexity of friction behaviors, experimental approaches seem only reliable solutions. Maru et al. [28] conducted the experiments on wear and friction responses of lubricated sliding tests. Fenz [29] experimented on the frictional properties of several low-friction, non-metallic materials in contact with stainless steel, and the dynamic coefficients of friction and their relationships with sliding velocity and normal pressure. Jaffer [27] developed a tribology facility which was capable of evaluating steady state friction and static friction properties of any material pair both in air and at high vacuum pressures. Thirteen material pairs were evaluated to determine sliding frictions a function of speed, load, distance, and test 
atmosphere. Based on the test results, Samyn et al. [30] and Sonne et al. [18] provided the correlation between the friction coefficient and contact pressure for low sliding velocity $(\mathrm{V}<0.6 \mathrm{~m} / \mathrm{s})$ - the resulting empirical, power-law equation is

$$
\mu=C p^{-n}
$$

where $p$ is the contact pressure and $\mathrm{C}=0.4$ and $n=0.63$.

\subsection{Numerical simulations}

Despite intensive research carried out over many years, the study of friction is still based on a number of hypotheses, each taking into account of individual and isolated interactions between sliding bodies. As far as hardness and roughness were concerned, Wieleba [31] modeled the friction under the dry condition between the PTFE and steel plate. He suggested that the roughness parameters related to the shape of asperities of the profile have strongest influence on the coefficient of friction, while the parameters related to the height of asperities have the strongest influence on the wear of PTFE.

Shi et al. [24] developed a deterministic mixed lubrication model for the interface between a moving smooth rigid surface and a stationary rough elastic surface. In general, a mixed lubrication model contains several coupled sub-models: (1) a flow model, to study the effect of a rough surface on the lubricant flow; (2) an elastic model, to analyze the structure distortion and surface deformation; and (3) a rough surface contact model, to determine the load sharing between hydrodynamic forces and surface contact forces. An elasto-hydrodynamic model consists of a hydrodynamic analysis of the flow in the lubricating film coupled with a deformation analysis of the lip material. The former produces the solutions for the pressure and shear stress distributions, which determines the deformation of the lip. The deformation analysis produces the film thickness distribution which affects the hydrodynamics.

Shen et al. [25] emphasized the transient condition such as startup and shutdown during which the fluid film is not fully established or it breaks down, and the seal operates in the mixed lubrication regime. A transient mixed lubrication analysis has been developed with consideration of load support, contact pressure, contact and cavitation ratio, reverse pumping rate, and average film thickness. A more realistic shaft surface with asperities is used, and the effect of the shaft surface roughness on the behavior of the seal is investigated. Many researchers have made important contributions to the theoretical and numerical analysis of mixed lubrication modeling. Benedetto et al. [32] extended Salant's approach to predict the lubricated conditions of radial lip seals; they took into account of normal and tangential deformation on the surface. Elastic influence coefficients are determined which are used to correct the surface deformation due to the lubricated film and hydrodynamic pressure. The film thickness is obtained by solving the Reynolds equations with consideration of the elastic deformation of surface. A linear perturbation method is used to predict the changes in deformation due to the changes in pressures caused by hydrodynamic effects.

Suisse et al. [33] suggested that for non-linear elasto-viscoplastic material, the history-dependent seal mounting process should be followed in finite element analysis (FEA). A new FEA procedure was presented to accommodate this need, which was based on a modified iterative 'rezoning' procedure. In many cases wear can be negligible if the lubrication is clean, but not in the field operation when the lubrication contains small particles. Rocke et al. [26] developed a numerical model incorporating the fluid mechanism of the lubricating film and the elastic deformation of the lip. Instead of treating asperities deterministically which required much computation, asperities were treated statistically. Cavitation and asperity orientation are taken into accounts in the computation of flow factors. This study showed that the operational parameters of seal and the characteristics of the asperities affect such seal characteristics as the thickness of lubricating film, reverse pumping rate, power dissipation, and liftoff speed. Suisse [33] developed physics-based analytic model for dynamic seal friction as a function of cylinder pressure, seal material, piston rod dimensions, piston rod seal gland dimensions, and other influencing factors. FEA was used to predict contact stress between the seal and the gland. The normal stress was used to determine coefficients of the test specimen. 


\subsection{Limitations of existing works}

It is unanimously agreed that friction involves a complex interaction of materials that is not easily quantifiable.

- Experimental approaches have the advantages of reliability. However, limited test data often cannot reveal the inherit relations between design variables and friction; moreover, the conditions in actual applications vary from the test conditions. It is impractical to do experiments for many applications and many changes - this requires test facilities and is very time-consuming.

- Friction behavior is very complicated, which involves multi-disciplines including fluid dynamics, deformation mechanics, and contact mechanics. While the governing equations have been developed in individual disciplines, it turns into a big challenge to integrate these governing equations for a complete solution of the friction model. All of existing numerical methods require an iterative solving process from the model in one discipline to that in another. Yet, the decomposition of an integrated model into individual sub-models in disciplines needs numerous assumptions, which are valid only to certain situations. These models have yet been verified for a wide application except for the seal-on-disk under a relatively high-speed. No analytical or numerical solution has been found for pin-to-plate under the conditions of the external load, the lubrication at contact, and boundary-type friction.

\subsection{Our work and organization of paper}

The focus of our work is to predict the friction force on pin-to-reciprocating plate under the specified lab condition and to verify the simulation model appropriately. In particular, the relative sliding motion is low, the contact happens between two flat surfaces, the type of friction is likely boundary type of friction. Most of existing works were developed for the pinon-disk configuration and the seal is lifted by the dynamic force; no work has been found on analytical model of friction at pin-to-plate contact under such specified conditions. A new approach has been developed to estimate the friction force.
The rest of the paper is organized as follows. In Section 2, a flow simulation is conducted under a typical test condition and pin geometry to confirm the lifting force and torque is insignificant in the specified range of sliding velocity. In Section 3, the impact of pin geometry (chamfer) on stress distribution is investigated; it is found the chamfer varies the stress distribution greatly. In Section 4 , a new friction model for the mixed boundary friction condition has been developed to quantify the impact of sliding velocity and pressure on friction. In Section 5, the numerical simulation has been established to integrate FEA with the friction model for the predication of friction; a discussion on the creditability of the developed friction model is provided. In Section 6, our work has been summarized.

\section{Lifting forces and torques from fluid flow}

The net friction force depends on the magnitude of the normal load and the coefficient of friction (COF). Since COF is indirectly measured by net friction force. It is necessary to identify any source which can alter the normal load of part significantly. One factor is the fluid flow in the test; the fluid in motion generates the forces and torques on walls. The free body diagram of the specimen is illustrated in Fig. 2. The lifting forces from fluid flow are $F_{x}$ and $F_{y}$ along $X$ and $Y$, respectively and the torque along $Z$ is $M_{z} . N$ is the applied load on the pin, $u_{x}$ is the average sliding velocity of reciprocating plate, $N^{\prime}$ is reaction force from plate to the pin, and $N_{\mathrm{L}}$ and $N_{\mathrm{R}}$ are the reaction forces from the pin to the fixture.

Assuming the friction between the fixture and pin is negligible, the force balances over the $\mathrm{XY}$ plane are

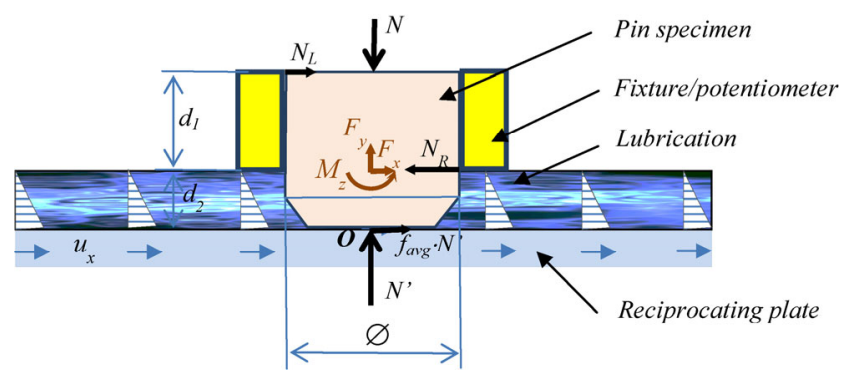

Fig. 2 Free-body diagram of pin specimen in test. 


$$
\left.\begin{array}{l}
\sum F_{x}=N-F_{y}-N^{\prime}=0 \\
\sum F_{y}=N_{L}+F_{x}+f_{\text {avg }} N^{\prime}-N_{R}=0 \\
\sum M_{z}=N_{L}\left(d_{1}+d_{2}\right)+\left(F_{x}-N_{R}\right) d_{2}-M_{Z}=0
\end{array}\right\}
$$

Equation (4) can further be used to find the relation of measured friction force with $F_{x}, F_{y}$ and $M_{z}$ from fluid flow as

$$
F_{\text {measure }}=N_{R}-N_{L}=F_{x}+f_{\text {avg }}\left(N-F_{y}\right)=f_{\text {avg }} N+\left(F_{x}-f_{\text {avg }} F_{y}\right)
$$

Therefore, $F_{x}$ will increase the measured friction force while $F_{y}$ will decrease the measured friction force. Since the reaction forces and torques over the bounded walls depend on the velocity of fluid flow, the maximum velocity of $16 \mathrm{ft} / \mathrm{min}$ in the test condition is used to investigate its impact on the measured friction force. The lubrication is specified as Tonna V68 with $\rho=880 \mathrm{~kg} / \mathrm{m}^{3}, v=68 \mathrm{~mm}^{2} / \mathrm{s}, \mu=0.05984 \mathrm{~kg} /(\mathrm{m} \cdot \mathrm{s})$ [34]. Flow simulation results for pressure distribution and vorticity are shown in Fig. 3 and Fig. 4, respectively.

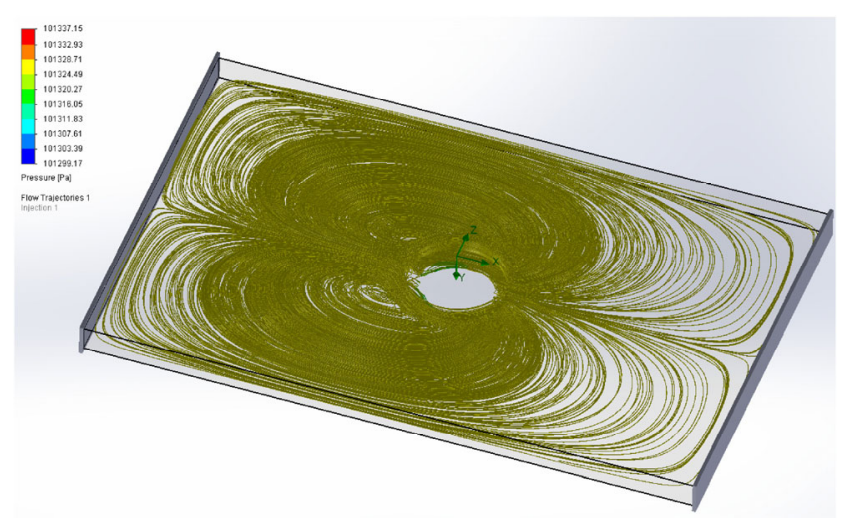

Fig. 3 Pressure distribution for $v=0.08333 \mathrm{~m} / \mathrm{s}$ in Tonna V68.

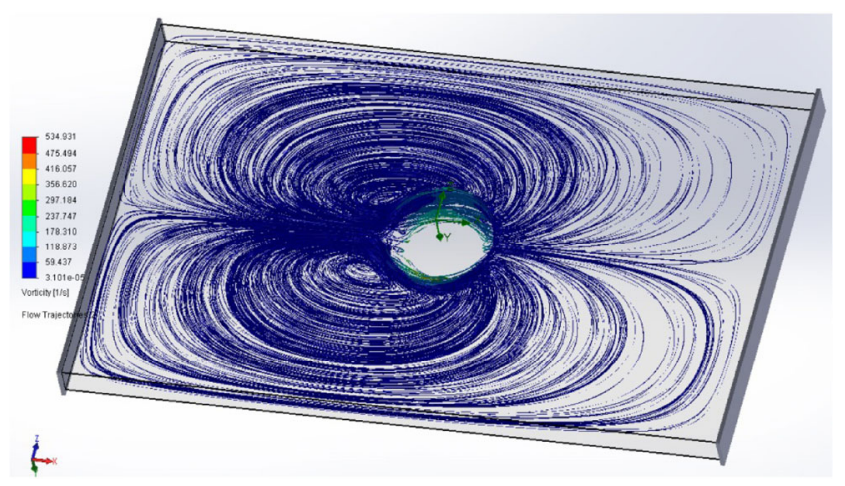

Fig. 4 Vorticity distribution for $v=0.08333 \mathrm{~m} / \mathrm{s}$ in Tonna V68.
Specifying all surfaces of pin under the fluid bed, the reaction forces and torques from the flow simulation are given in Table 2. Therefore, under a given load $N=$ $88.964 \mathrm{~N}$ in the test, the lifting forces and torques have a little impact on the measured friction coefficient.

\section{Stress distribution vs. chamfer angle}

It is well accepted that COF varies with the pressure. In other words, COF is different from one position to another based on the level of normal stress at the position. It is critical to determine the stress distribution under the given load. In this section, the impact on chamfer angle on the stress distribution is considered using FEA. Given the external load of $20 \mathrm{lbf}$, the stress on the bottom of the pin is calculated with the chamfer angle of $0.5^{\circ}, 5^{\circ}, 10^{\circ}, 15^{\circ}, 20^{\circ}, 25^{\circ}, 30^{\circ}, 35^{\circ}, 40^{\circ}$, and $90^{\circ}$. Note that the case of $90^{\circ}$ corresponds to the same diameter of pin bottom. As illustrated in Fig. 5 when chamfer angle is $20^{\circ}$, the pin chamfer causes the stress concentration over the edge. The further analysis of FEA data has shown that the normal stress at one position is monotonically increased with its distance to the pin center in Fig. 6.

The external load is balanced by the normal reaction forces on the pin bottom, i.e., a higher normal stress

Table 2 Lifting forces and torques from fluid flow.

\begin{tabular}{cccc}
\hline Goal name & Unit & Value & Criteria \\
\hline SG force $(X) 1$ & $\mathrm{~N}$ & $2.66076 \mathrm{E}-05$ & $2.16689 \mathrm{E}-06$ \\
SG force $(\mathrm{Y}) 1$ & $\mathrm{~N}$ & $1.8711 \mathrm{E}-05$ & $4.30619 \mathrm{E}-07$ \\
SG force $(\mathrm{Z}) 1$ & $\mathrm{~N}$ & $6.7499 \mathrm{E}-08$ & $4.29281 \mathrm{E}-08$ \\
SG torque $(\mathrm{X}) 1$ & $\mathrm{~N} \cdot \mathrm{m}$ & $-1.54886 \mathrm{E}-09$ & $1.82831 \mathrm{E}-10$ \\
SG torque $(\mathrm{Y}) 1$ & $\mathrm{~N} \cdot \mathrm{m}$ & $-2.2599 \mathrm{E}-09$ & $2.96267 \mathrm{E}-10$ \\
SG torque $(\mathrm{Z}) 1$ & $\mathrm{~N} \cdot \mathrm{m}$ & $-2.69961 \mathrm{E}-07$ & $5.24023 \mathrm{E}-09$ \\
\hline
\end{tabular}

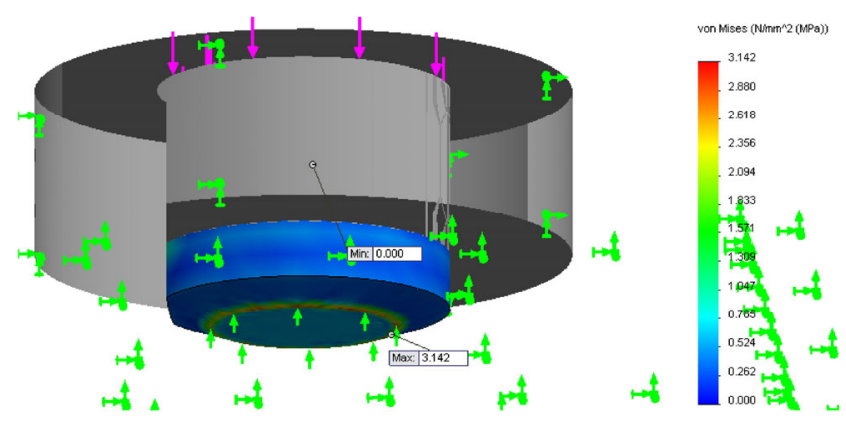

Fig. 5 Stress distribution over contact area (load $=20 \mathrm{lbf}$ and chamfer angle $=20^{\circ}$ ). 
implies a greater normal reaction force. Figure 7 and Fig. 8 show the normal reaction force and its percentage of the load share, respectively.

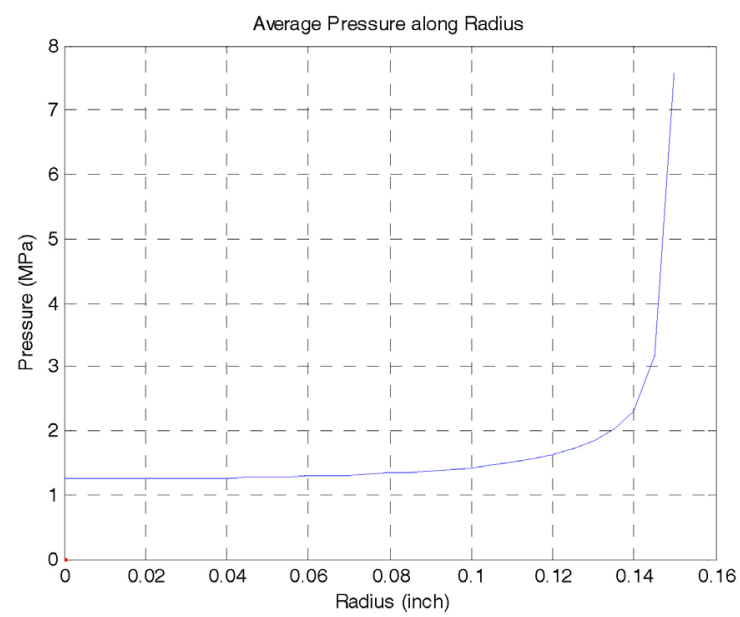

Fig. 6 Normal stress along radius direction ( chamfer angle $=20^{\circ}$ ).

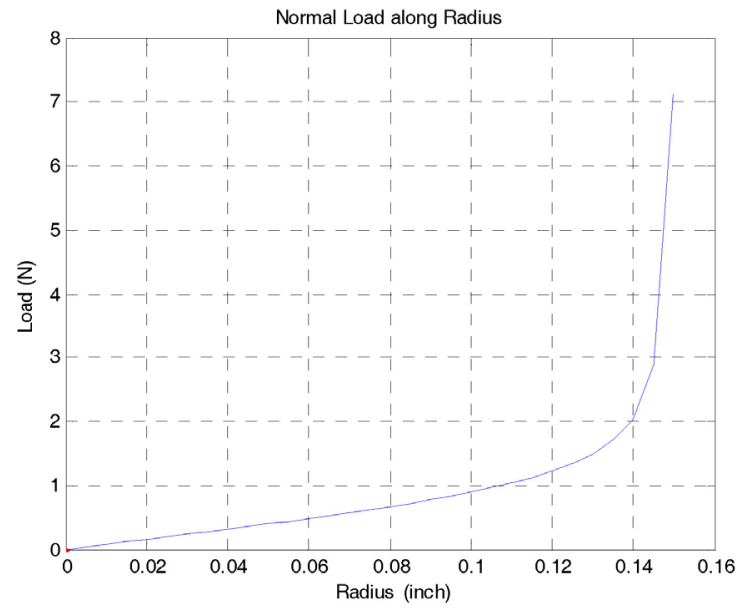

Fig. 7 Normal load along radius direction ( chamfer angle $\left.=20^{\circ}\right)$.

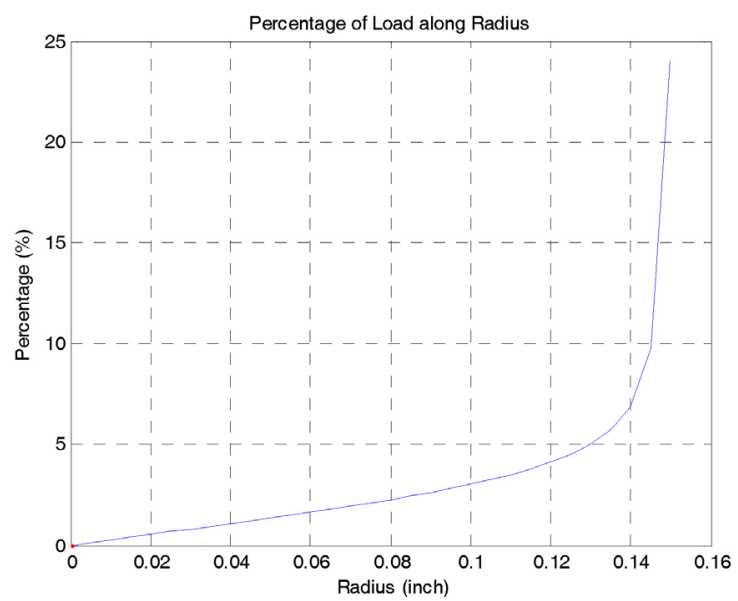

Fig. 8 Percentage of load share along radius direction (chamfer angle $=20^{\circ}$ ).
Figures $6-8$ show that the stress contrenstration by the chamfer angle causes the area close to the edge carries more load than the central area. Therefore, the relation of stress contrentration and chamfer in Fig. 9 is important to under the impact of chamfer angle on friction.

\section{Modeling of pin-to-plate friction}

\subsection{Pin-plate contact}

As shown in Fig. 10, the following parameters or assumptions are made in developing the friction model at the pin-to-plate contact: (1) the pin and the plate are under a low relative motion with the range of $(5-5000 \mathrm{~mm} / \mathrm{min})$; (2) there is an external load applied on the pin, and the load is sufficient to overcome fluid pressure so that the pin always contacts to the reciprocating metal plate; (3) the level of fluid is low in contrast to the height of the pin; the combination of the fluid pressure and velocity is insufficient to lift pin. Under such assumptions, the contact area consists of fluid cavities and contacted asperities, and the friction between the pin and reciprocating metal plate belongs to the mixed boundary friction.

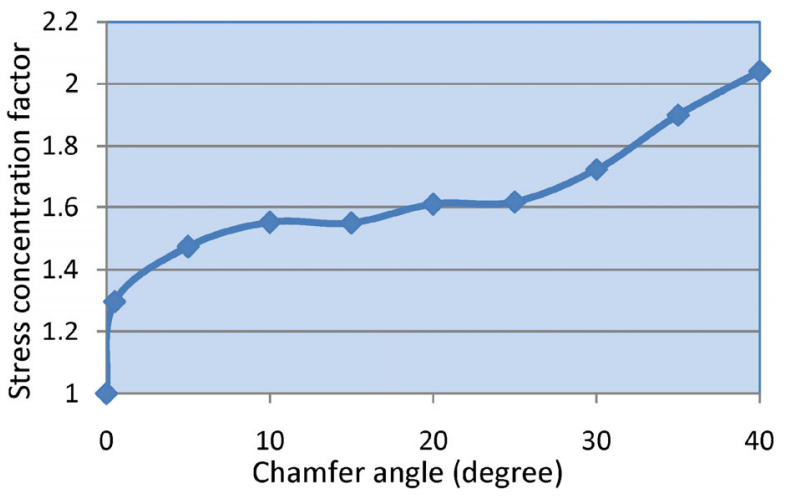

Fig. 9 Stress concentration vs. chamfer angle.

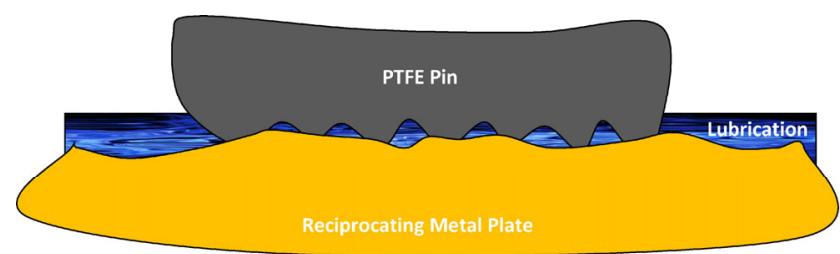

Fig. 10 Pin-to-plate interface with fluid cavities and contact asperities. 
The total contact area consists of (a) the area of fluid cavities, and (b) the area of the deformed asperities as

$$
A_{\text {total }}=A_{\text {fluid }}+A_{\text {contact }}
$$

where $A_{\text {total }}$ is total area of the contact at the flat bottom of the pin; $A_{\text {fluid }}$ is the summed area of the fluid cavities; $A_{\text {contact }}$ is the summed area of deformed asperities.

\subsection{Force balance along load direction}

Revisiting the free-body diagram in Fig. 2, the net force along the load direction of the pin must be balanced; i.e., the external force equals to a sum of the normal reaction forces gathered at the area of the cavities and the area of the asperities

$$
N_{\text {total }}=p_{\text {fluid }} A_{\text {fluid }}+p_{\text {contact }} A_{\text {contact }}
$$

where $N_{\text {total }}$ is external normal load over pin, $p_{\text {fluid }}$ is average fluid pressure in cavities, $p_{\text {contact }}$ is average normal stress at deformed asperities.

\subsection{Object deformation under pressure}

The friction coefficient is different at the cavities and contacted asperities. In evaluating the friction force, the critical challenge is to determine the ratio of area of fluid cavities and that of deformed asperities. No literature has been found on this issue.

In this section, we assume that the ratio of area relates of the pressure (or normal stress) at certain area. As shown in Fig. 11, two extreme cases can be identified: (1) when the pressure at the certain area is less than ambient fluid pressure, the pin is lifted. There will be a layer of the fluid film, and all of the area at bottom of pin is the fluid area; (2) when the pressure at certain area is larger than the yield strength

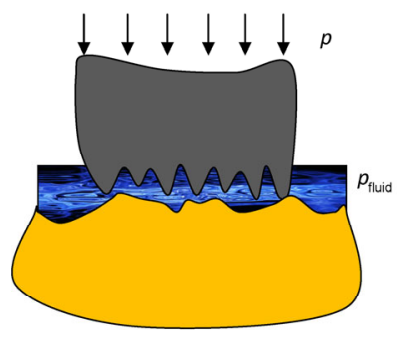

(a) Full fluid area when $p<p_{\text {fluid }}\left(R_{c}=0\right)$

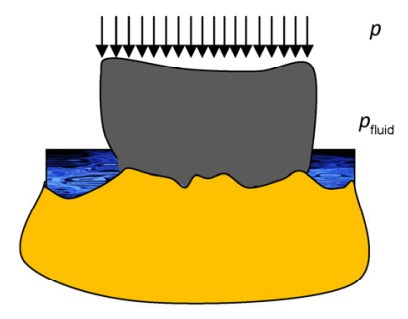

(b) Full asperities area when $p>S_{y}\left(R_{c}=1\right)$
Fig. 11 Extreme cases of the ratio of fluid area and asperities area. of pin material, yielding happens everywhere thus all of the area is with deformed asperities.

The exact area ratio is impossible to be quantified due to the randomness and roughness on the contact areas. However, this ratio is directly related to the deformation of asperities of materials; i.e., when the pressure over the area is increased, more and more asperities are deformed to carry the load. Theoretically, the deformation of the asperities transfers the external energy into the strain energy which can be defined as follows

$$
U_{p}=\int \frac{\sigma \varepsilon}{2} d V \cong \frac{p_{\text {avg }}^{2}}{2 E} V_{p}
$$

where $U_{p}$ is the strain energy caused by the deformation of asperities, $\sigma$ and $\varepsilon$ are the stress and strain at an arbitrary point in the deformed asperities, $p_{\text {avg }}$ is an equivalent pressure to cause the deformation of the asperities, $V_{p}$ is a total volume of the deformed asperities under external pressure $p_{\text {avg }}$.

Therefore, given the strain energy under a given pressure, Eq. (8) can be transferred into

$$
V_{p}=\frac{2 E U_{p}}{p_{\mathrm{avg}}^{2}}
$$

Note that our objective is to determine the area ratio. Equation (9) leaves us the clue that the volume of deformed asperities is inversely proportional to the square of $p_{\text {avg }}$. Therefore, the area ratio is assumed to be given by

$$
\begin{aligned}
R_{c} & =\frac{V_{\text {contact }}}{V_{\text {total }}}=\frac{A_{\text {contact }}}{A_{\text {total }}} \\
& = \begin{cases}0, & p_{\text {contact }} \leq 0 ; \\
{\left[1-\cos \pi\left(\frac{p_{\text {contact }}}{S_{y}}\right)^{2}\right] / 2,} & 0<p_{\text {contact }}<S_{y} ; \\
1 . & p_{\text {contact }} \geq S_{y}\end{cases}
\end{aligned}
$$

where $R_{c}$ is the ratio of the area of cavities and the area of deformed asperities. Equation (10) can be graphically depicted in Fig. 12. The area ratio is 0 when $p_{\text {avg }}$ is less than $p_{\text {fluid, }}$ the increase of $p_{\text {avg }}$ increases the ratio until $p_{\text {avg }}$ reaches $S_{y}$ when the ratio is 1 . 


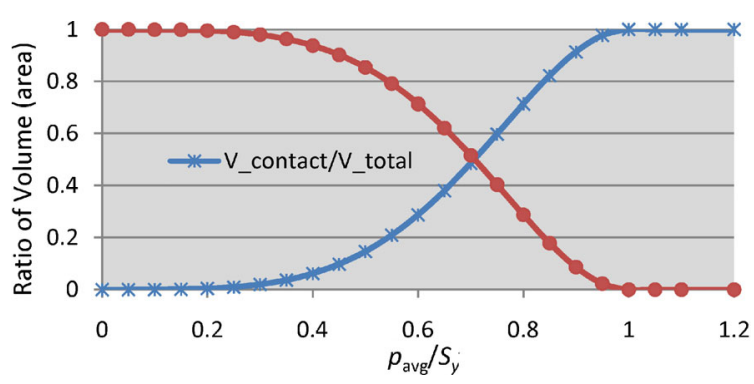

Fig. 12 Ratio of fluid area and asperities area versus $p_{\text {avg. }}$.

\subsection{Fluid pressure loss in cavities}

Since the specimen is immersed in the lubrication, the cavities within the contact area capture oil and the oil pressure can be sustained if the sliding velocity is lower; otherwise, the oil can be leaked if the sliding velocity is higher. As shown in Fig. 13, since the oil pressure carries the normal load as well; it is necessary to develop the relation of the pressure loss and sliding velocity as well. Therefore, the friction coefficient for fluid flow under laminar conditions is expressed as [35]

$$
\lambda=64 / R_{e}=\frac{64 \mu}{d \cdot v \cdot \rho}=\frac{64 v}{d \cdot v}
$$

where $\lambda$ is the friction coefficient (nondimensional), $R_{e}$ is the Reynolds number (nondimensional), $\mu$ is the absolute viscosity $(\mathrm{kg} / \mathrm{m}-\mathrm{s}), d$ is the internal diameter $(\mathrm{m})$; in this case study corresponds to average roughness, $v$ is the mean velocity of flow $(\mathrm{m} / \mathrm{s}), \rho$ is the mass density of fluid $\left(\mathrm{kg} / \mathrm{m}^{3}\right), v$ is the $\mu / \rho$ is kinematic viscosity $\left(\mathrm{m}^{2} / \mathrm{s}\right)$.

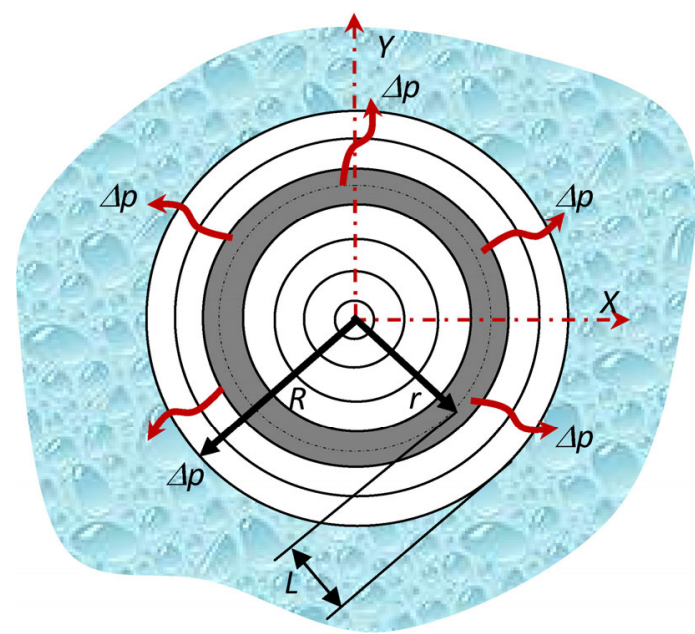

Fig. 13 Pressure loss in cavities due to fluid flow.
Assume the fluid flow is laminar, thus the pressure drop can be estimated as [36]

$$
\Delta p=\frac{32 \mu v L}{d^{2}}
$$

where $\Delta p=$ pressure drop $\left(\mathrm{N} / \mathrm{m}^{2}\right), L$ is the distance from the interested position to the fluid bed.

As shown in Fig. 13, $L$ can be estimated as $L=R-r$, where $R$ is the pin diameter and $r$ is the radius the ring is located.

\subsection{Determination of contact pressure}

Revisiting Eq. (5) again, the following constraint has to be satisfied to achieve the force balances along the load direction simultaneously,

$$
p_{\text {avg }} A_{\text {total }}=\left(p_{\text {avg }}-\Delta p\right)\left(1-R_{c}\right) A_{\text {total }}+p_{\text {contact }} R_{c} A_{\text {total }}
$$

Then the average pressure at deformed asperities is

$$
p_{\text {contact }}=\frac{p_{\text {avg }}-\left(p_{\text {avg }}-\Delta p\right)\left(1-R_{c}\right)}{R_{c}}
$$

An iterative process can be applied to find both of $R_{c}$ and $p_{\text {contact }}$ from Eqs. (10) and (14).

\subsection{Dynamic friction}

Under an extreme low velocity, not all contacts of asperities are dynamic friction. As illustrated in Fig.14, an asperity is deformed plastically only when an axial load exceeds the critical buckling load; when the pressure is below the buckling load, the asperity may be deformed elastically without a relative motion at the contact even there is a relative motion between the pin and sliding plate. The friction at such asperities is static which varies from 0 to the magnitude of dynamic COF. To take into consideration of this aspect, the area of asperities contact is divided into the area with a dynamic friction and the area with a static friction. The contact at an asperity has a dynamic friction only when (1) the normal pressure is high enough to cause the buckling of the asperity; or (2) the sliding velocity is high enough to prevent the deformed asperity return to the elastic status, i.e., the time to cause the shear stress at the contact is smaller than a quart of natural frequency of asperity body. 


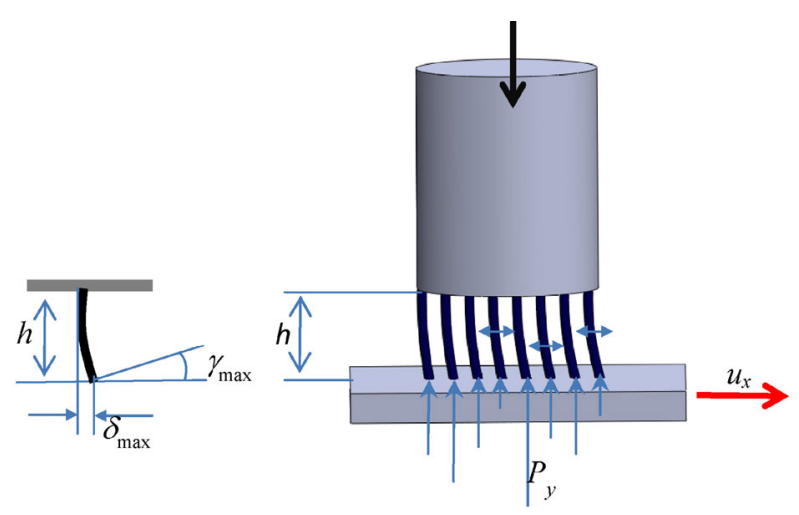

Fig. 14 Simplified model of asperities.

Assume an asperity is a round column fixed at one end and supporting an axial load at the other end. Its natural frequency can be found as [37]

$$
\omega_{n}=\alpha_{n}^{2} \sqrt{\frac{E I}{\rho A L^{4}}}
$$

where $\omega_{n}$ is the $n^{\text {th }}$ natural angular velocity with $\alpha_{n}=$ $1.875,4.694$, and 7.885 for the first three modes of vibrations.

As shown in Fig. 14, the travel distance for an asperity to return its home position is

$$
\delta_{\text {max }}=h \tan \gamma_{\text {max }} \cong h \gamma_{\text {max }}
$$

Therefore, the second condition can be represented as

$$
t=\frac{4 \delta_{\max }}{u_{x}} \leq \frac{1}{f}=\frac{2 \pi}{\omega_{1}}
$$

Assume the minimal velocity to have a sufficient time traveling back to a home position $u_{\min }=4 f \delta_{\max }$, the percentage of asperities under dynamic friction can be estimated by

$$
R_{d y n a}=\frac{u_{x}}{u_{\min }}
$$

The sliding speed changes the proportion of two areas until it reaches the maximum that all contact areas happened to have the permanent deflection and the dynamic friction.

\subsection{Estimation of friction force}

As a result, the friction force in Eq. (6) can be further expressed as

$$
\begin{aligned}
F_{\text {total }} \approx & f_{\text {avg }} N=\mu \frac{\partial u}{\partial y}\left(1-R_{c}\right) A_{\text {total }} \\
& +\frac{\left(1+R_{\text {dyna }}\right) f_{\text {dry }}}{2} p_{\text {contact }} R_{c} A_{\text {total }}
\end{aligned}
$$

where $F_{\text {total }}$ is the total friction force over the given area, $A_{\text {total }}$ denotes a small area with an average load pressure of $p_{\text {avg }} \partial u / \partial y$ is the gradient of velocity along the load direction, $R_{c}$ and $p_{\text {contact }}$ are defined by Eqs. (10) and (14), $f_{\text {dry }}$ is the COF under the dry sliding condition, $R_{\text {dyna }}$ is the ratio of dynamic friction area define in Eq. (18), $f_{\text {avg }}$ is the average COF relating to $p_{\text {contact }}$ and sliding velocity $u_{x}$.

\section{Numerical simulation to predict friction force}

It is well known that the friction behavior is very complicated. Besides major factors such as contact pressure and relative motion velocity, there are many other factors such as the roughness, the pattern and texture of contact surfaces, as well as the temperature and viscosity of the fluid. In this study, only the impacts of geometric change of a pin, sliding velocity, and pressure are considered as design variables while the other factors are fixed. Based on the developed model in Section 4, the procedure for numerical simulation in Fig. 15 has been developed to predict COF.

As shown in Fig. 15, some basic information, such as a COF under the dry friction condition should be acquired from a minimal number of tests. Some parameters used in the simulation, such as the material properties of PTFE, the roughness and pattern, and the minimal velocity to reach the complete dynamic friction, should be measured before the simulation. Each numerical simulation can be used to predict one $\mathrm{COF}$ for one set of variables. Therefore, multiple loops of the simulations are required when more than one design variables are investigated. In the flowchart, the velocities and chamfer angles are treated as design variables. Please note that due to the randomness and uncertain factors of tests and numerous simplifications in modeling, the results of numerical 
simulation can only be used to explain the friction phenomenon qualitatively rather than quantifiably.

As a case study, the inputs in Table 3 are applied.

Note that the COF $\left(f_{\text {dry }}\right)$ under the dry condition and the minimal velocity $u_{\min }$ for dynamic friction have been estimated based on the test data in Fig. 16 available at the Trelleborg website [13].

As a result, the COF for the pin chamfer angle $20^{\circ}$ of has been found in Fig. 17. It can be seen that the COF changes dramatically even within a small

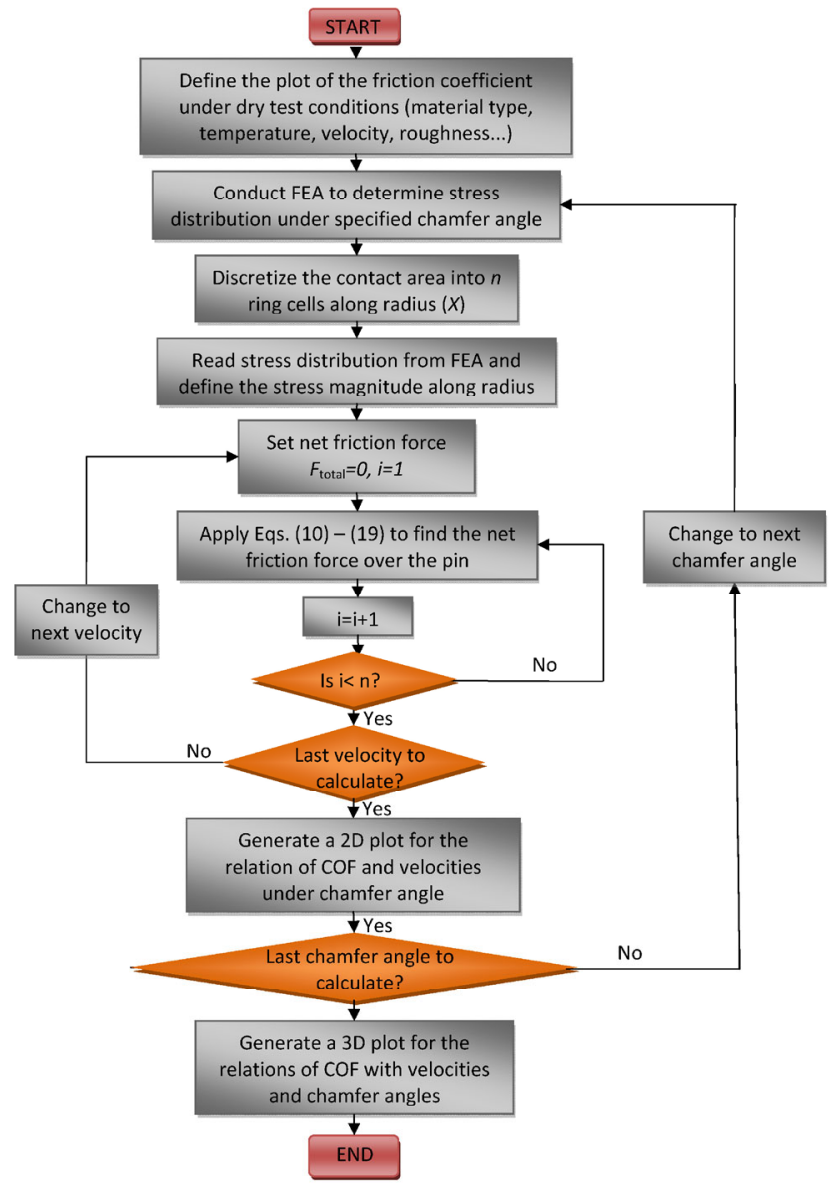

Fig. 15 Numerical simulation to predict COF under given load.

Table 3 Initialization of variables in simulation.

\begin{tabular}{ccc}
\hline \multirow{2}{*}{ Lubrication } & Density & $880 \mathrm{~kg} / \mathrm{m}^{3}$ \\
(Tonna V68) & Dynamic viscosity & $0.059840 \mathrm{~kg} /(\mathrm{m}-\mathrm{s})$ \\
& Kinematic viscosity & $68 \mathrm{~mm}^{2} / \mathrm{s}$ \\
& Load & $20 \mathrm{lbf}$ \\
Specimen & Roughness & $0.3 \mu \mathrm{m}$ \\
(PTFE) & Velocity & $5-50,000 \mathrm{~mm} / \mathrm{min}$ \\
& $f_{\text {dry }}$ & 0.071246 \\
& $u_{\min }$ & $0.26927 \mathrm{~m} / \mathrm{s}$ \\
\hline
\end{tabular}

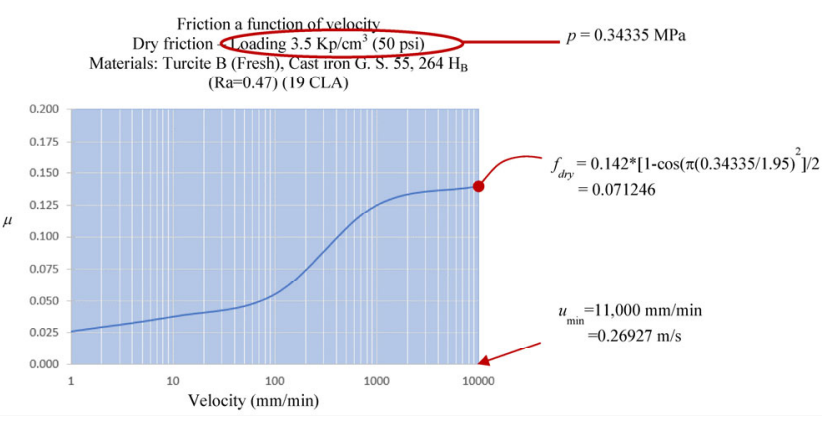

Fig. 16 COF under dry friction condition for PTFE [13].

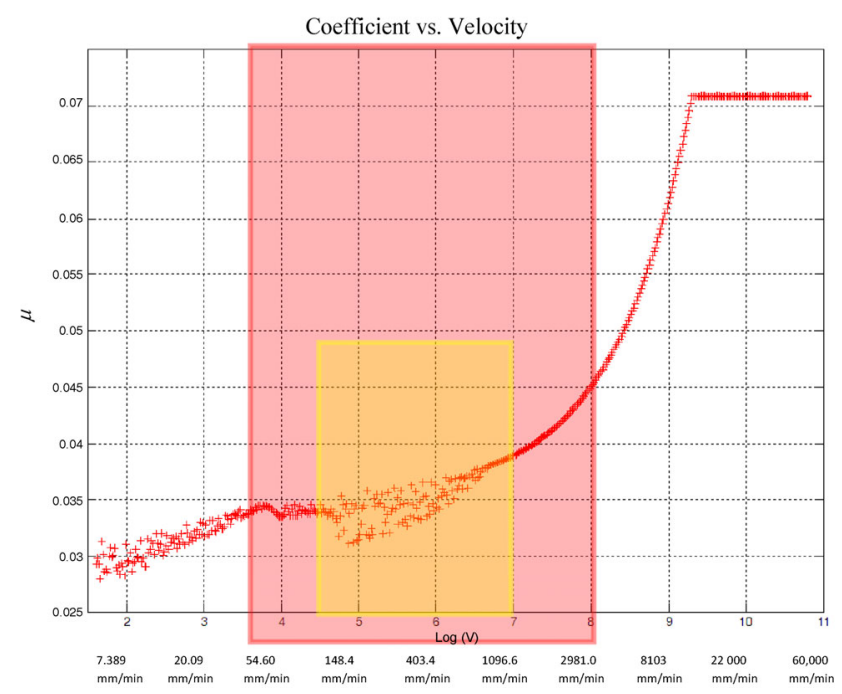

Fig. 17 Predicted COF under $20 \mathrm{lbf}$ load with pin chamfer angle of $20^{\circ}$.

distribution of velocity, e.g., $50-1,000 \mathrm{~mm} / \mathrm{min}$. Generally, COF increases gradually with the increase of the sliding velocity until it reaches a minimal magnitude for a full region of the dynamic friction. A further comparison with the data in Fig. 18 from Trelleborg [13] might show similar variation of the COF despite the fact that the simulation result has shown oscillation within the range of low velocity from 50 to $1,000 \mathrm{~mm} / \mathrm{min}$. We believe it is caused by the simplification in assessing the pressure in cavities and the area of dynamic friction related to sliding velocity. By all means, the surface roughness is statistical average.

\section{Summary}

A comprehensive literature review has proven that the quantifiable approach to predict accurate COF under 


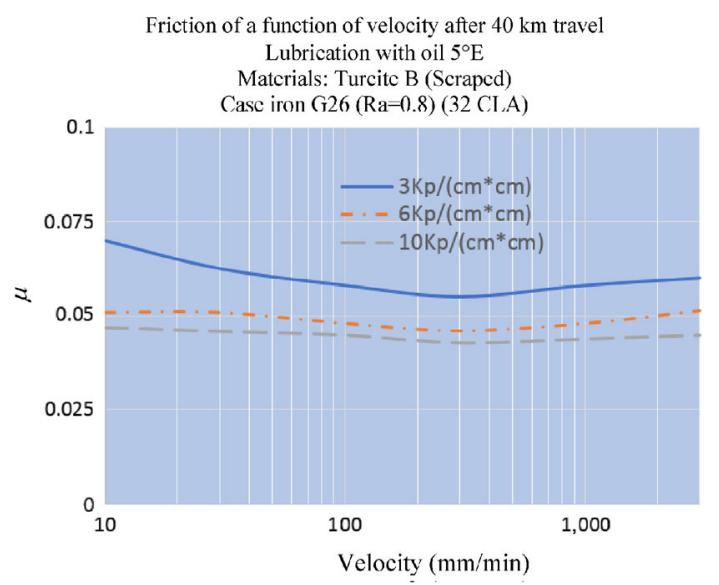

Fig. 18 COF under lubrication friction condition for PTFE [13].

variable conditions is very challenging. The main reason is that the friction behavior relates closely to the microstructure of contact surfaces; this is particularly true when the mixed boundary friction is considered. The microstructure of contact surfaces shows randomness and uncertainties which has to be represented statistically, while most of the governing equations in fluid dynamics, contact mechanics, and solid mechanics are developed based on deterministic variables and parameters.

To predict COF qualitatively, a novel friction model has been proposed and the procedure in applying such a model to estimate COF has been developed. The model is simplified by using deterministic variables instead of statistic variables; however, it has been applied to investigate the impact of different parameters such as external load (pressure) and sliding velocities on the COF. The following conclusions have been drawn preliminarily from the proposed model:

- Under the given test conditions and preferable ranges of variables, the flow simulation has shown that the lifting forces and torques from lubrication are insignificant can be neglected. The measured friction force is from the pin-to-plate contact. For the mixed boundary condition, the net friction force consists of shear forces at the fluid cavities and the friction forces at the deformed asperities of contact. Therefore, the friction force depends on (a) the magnitude of shear stress, the holding pressure and the area of fluid cavities and (b) the magnitude of normal pressure, the percentage of dynamic friction, and the area of deformed asperities.
- It is found that the pressure affects COF since it changes the area of deformed asperities at the contact area, and the percentage of the deformed asperities can be estimated based on the equivalent strain energy. The higher the pressure, the more asperities are deformed; when the pressure reaches yield strength, all of asperities are deformed. When the contact pressure is less than ambient pressure, the mixed boundary friction becomes fully hydrodynamic friction.

- The chamfer angle affects the COF since it causes the stress concentration close to the circle edge; the large angle it is, the higher the edge stress concentration. It is possible to compare COFs for different chamfer angles if other parameters such as sliding velocities and surface roughness are given. However, the stress distribution over a certain area changes all the factors relating to friction forces, the impact of chamfer angle on COF is not monotonic.

- The sliding velocity affects the pressure drops of the cavities greatly. Since the fluid flow in the test condition is laminar, the lower the sliding velocity, the higher normal pressure the cavities can sustain. The cavity pressure shares the normal load so that the friction at deformed asperities can be reduced. When the sliding velocity reaches a certain level, there is no difference between pressure in cavities and in ambient environment; all external load is carried by deformed asperities. However, the quantification of pressure drops of cavities is difficult since the randomness of surface conditions. The proposed model is simplified by used deterministic variables for geometries of fluid flow.

- The sliding velocity affects the area of dynamic friction at the deformed asperities as well. When the sliding velocity is very low, not all of deformed asperities experience dynamic friction. The appearance of static frictions within the contact areas can generally reduce the friction at those contacts. Further, it is assumed that there is a minimal sliding velocity where all of the deformed asperities experience dynamic friction. The percentage of the area with the dynamic friction has been quantified based on (a) the pressure level to cause the buckling of asperitiesand (b) a comparison of the travel time for an elastic asperity to return its home position and its natural frequency. 
- All of the proposed models have been integrated to implement the predicting process; it can be refined to better fulfill its capabilities with a reasonable effort as long as the reliable raw data can be obtained from a minimal set of tests.

\section{Acknowledgements}

The first author would like to acknowledge the support by the State International Science and Technology Cooperation Special Items (Grant No. 2015DFA11700), the Frontier and Key Technology Innovation Special Funds of Guangdong Province (Grant Nos. 2014B090919002 and 2015B010917003), and the Program of Foshan Innovation Team of Science and Technology (Grant No. 2015IT100072).

Open Access: The articles published in this journal are distributed under the terms of the Creative Commons Attribution 4.0 International License (http:// creativecommons.org/licenses/by/4.0/), which permits unrestricted use, distribution, and reproduction in any medium, provided you give appropriate credit to the original author(s) and the source, provide a link to the Creative Commons license, and indicate if changes were made.

\section{References}

[1] Persson B N J. Sliding Friction. Surface Sci Rep 33(3): 83-119 (1999)

[2] Parker. Hydraulic seals.https://www.parker.com/literature/ Praedifa/Catalogs/Catalog_HydrSeals_PTD3350-EN.pdf, last accessed on April 11, 2018.

[3] Trelleborg Sealing Solution. Fluid power seal design guide catalog EPS 5370. https://www.parker.com/literature/ Engineered\%20Polymer\%20Systems/5370.pdf, last accessed on April 11, 2018.

[4] Dornfeld D A. Process modelling and control for precision manufacturing. In $15^{\text {th }}$ Brazilian Congress of Mechanical Engineering. Lindoia, Sao Paulo, http://www.abcm.org.br/ anais/cobem/1999/pdf/LecDornfeld_S19.pdf, 1999.

[5] Cochran D S, Hendricks S, Barnes J, BiZ M. Extension of manufacturing system design decomposition to implement manufacturing systems that are sustainable. J Manuf Sci Eng 138(10): 101006 (2016)

[6] Bi Z M, Xu L D, Wang C E. Internet of things for enterprise systems of modern manufacturing. IEEE Trans Ind Inf 10(2): 1537-1546 (2014)

[7] Li J W, Chen X B, An Q, Tu S D, Zhang W J. Friction models incorporating thermal effects in highly precision actuators. Rev Sci Instrum 80(4): 045104 (2009)

[8] Zhang Q S, ChenX B, Yang Q, Zhang W J. Development and characterization of a novel piezoelectric-driven stickslip actuator with anisotropic-friction surfaces. Int $J A d v$ Manuf Technol 61(9-12): 1029-1034 (2012)

[9] Zhang Z M, An Q, Li J W, Zhang W J. Piezoelectric friction-inertia actuator-a critical review and future perspective. Int J Adv Manuf Technol 62(5-8): 669-685 (2012)

[10] Liu Y F, Li J, Hu X H, Zhang Z M, Cheng L, Lin Y, Zhang W J. Modeling and control of piezoelectric inertia-friction actuators: review and future research directions. Mech Sci 6(2): 95-107 (2015)

[11] Liu Y F, Li J, Zhang Z M, Hu X H, Zhang J. Experimental comparison of five friction models on the same test-bed of the micro stick-slip motion system. Mech Sci 6(1): 15-28 (2015)

[12] TrelleborgSealing Solution. Turcite ${ }^{\circledR}$ B Slydway ${ }^{\circledR}$ Technical Data. http://mtsandtg.com/system/ckeditor_assets/attachments/ 80/slydwayformtg.pdf, last accessed on April 11, 2018.

[13] WS Hampshire Inc. The properties of PTFE. http://www. wshampshire.com/data/psg_ptfe.pdf, 2018.

[14] Bearing Works. (PTFE) Polytetrafluoroethylene. http:// www.bearingworks.com/content_files/pdf/retainers/PTFE\% 20datasheet.pdf, 2018.

[15] ZEUS. Friction and wear of polymers. Technical Whitepaper, http://www.appstate.edu/ clementsjs/polymerproperties/ \$p\$lastics_\$f 11, 2018.

[16] Biswas S K, Vijayan K. Friction and wear of PTFE - A review. Wear 158(1-2): 193-211 (1992)

[17] Xie G Y, Zhuang G S, Sui G X, Yang R. Tribological behavior of PEEK/PTFE composites reinforced with potassium titanate whiskers. Wear 268(3-4): 424-430 (2010)

[18] Sonne M R, Hattel J H. Modeling the constitutive and frictional behavior of PTFE flexible stamps for nanoimprint lithography. Microelectron Eng 106: 1-8 (2013)

[19] Jagger E T. Rotary shaft seals: the sealing mechanism of synthetic rubber seals running at atmospheric pressure. Proc Instit Mech Eng 171(1): 597-616(1957)

[20] Kato K. Wear in relation to friction - A review. Wear 241(2): 151-157 (2000)

[21] Quaglini V, Dubini P. Friction of polymers sliding on smooth surfaces. Adv Tribol 2011: 178943 (2011)

[22] Stolarshi T. Tribology in Machine Design. Amsterdam: Elsevier Science, 2000. 
[23] Salant R F, Shen D W. Hydrodynamic effects of shaft surface finish on lip seal operation. Tribol Trans 45(3): 404-410(2002)

[24] Shi F H, Salant R F. A mixed soft elastohydrodynamic lubrication model with interasperity cavitation and surface shear deformation. J Tribol 122(1): 308-316(2000)

[25] Shen D W, Salant R F. A transient mixed lubrication model of a rotary lip seal with a rough shaft. Tribol Trans 49(4): 621-634 (2006)

[26] Rocke A H, Salant R F. Elastohydrodynamic analysis of a rotary lip seal using flow factors. Tribol Trans 48(3): 308-316 (2005)

[27] Jaffer F. Experimental evaluation of sliding friction coefficients for aerospace applications. MS Thesis. Toronto (Canada): University of Toronto, 2001.

[28] Maru M M, Tanaka D K. Consideration of stribeck diagram parameters in the investigation on wear and friction behavior in lubricated sliding. J Braz Soc Mech Sci Eng 29(1): 55-62 (2007)

[29] FenzD. Frictional properties of non-metallic materials for use in sliding bearing: an experimental study. Education, 113-118, http://mceer.buffalo.edu/publications/resaccom/02SP09/pdfs_screen/19_Fenz.pdf, 2009.

[30] Samyn P, Schoukens G. Experimental extrapolation model for friction and wear of polymers on different testing scales.

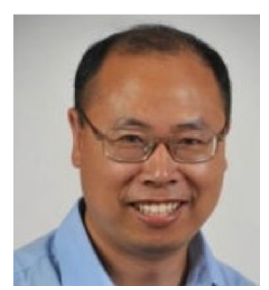

Zhuming BI. He received the bachelor degree in manufacturing engineering in 1987 from Harbin University of Science and Technology, the master of science in mechanical engineering and the $\mathrm{PhD}$ in mechatronic control and automation from Harbin Institute of Technology in 1991 and 1994, respectively.

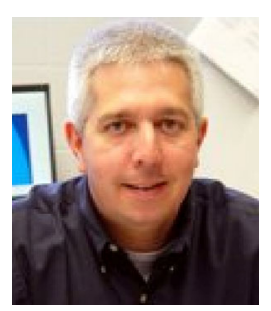

Donald W. MUELLER. He received all of his bachelor degree, master of Science, and $\mathrm{PhD}$ degree in mechanical engineering from University of Missouri-Rolla. He is
Int J Mech Sci 50(9): 1390-1403(2008)

[31] Wieleba W. The statistical correlation of the coefficient of friction and wear rate of PTFE composites with steel counterface roughness and hardness. Wear 252(9-10): 719-729 (2002)

[32] Benedetto G D, Organisciak M, Popovici G, Stijepic A. Film thickness prediction of radial lip seal. FME Trans 37(2): 87-90 (2009)

[33] Suisse B E. Research for dynamic seal friction modeling in linear motion hydraulic Piston applications. MS Thesis. Arlington: The University of Texas at Arlington, 2007.

[34] Shell Tonna Oils V. Technical Data Sheet. aglayne.com/ wp-content uploads/2010/11/Tonna-V-68.pdf, 2013.

[35] Engineering Toolbox. Friction coefficient at laminar flow. http:// www.engineeringtoolbox.com/laminar-frictioncoefficient-d_1032.html, last accessed on April 11, 2018.

[36] Rechtenwald G. Head loss in pipe systems, laminar flow and introduction to turbulent flow. http://web.cecs.pdx.edu/ $\sim$ gerry/class/ME322/notes/pdf/ME322_lect03slides.pdf, last accessed on April 11, 2018.

[37] Sakshat Virtual Labs. Free vibration of a cantilever beam. http://iitg.vlab.co.in/?sub=62\&brch=175\&sim=1080\& cnt=1, last accessed on April 11, 2018.

He received the second $\mathrm{PhD}$ degree in mechanical engineering from University of Saskatchewan in 2002. $\mathrm{He}$ is a professor of mechanical engineering in Purdue University Fort Wayne, and his research interests are modelling and simulation, manufacturing systems, robotics and automation.

an associate professor of mechanical engineering in Purdue University Fort Wayne, and his research interests are thermal sciences, machine design, and numerical methods. 